\section{Age related increase in the intra-alveolar macrophage population of non-smokers}

\author{
W A H Wallace, M Gillooly, D Lamb
}

\begin{abstract}
The alveolar macrophage population is higher in smokers than in non-smokers. An age related increase in the alveolar macrophage number in the lungs of nonsmokers has been found, whether expressed per unit lung volume or per unit lung surface area.
\end{abstract}

(Thorax 1993;48:668-669)

The intra-alveolar macrophage population has been shown to be increased in smokers compared with non-smokers on the basis of bronchoalveolar lavage ${ }^{1}$ and morphometric studies on tissue sections. ${ }^{2}$ No data are available, however, on changes in the alveolar macrophage population of non-smokers with age, despite recognised age related changes in lung structure and function. ${ }^{34}$

We have counted the alveolar macrophage number in a group of non-smokers using tissue sections and morphometric techniques. The results have been expressed per unit lung volume $\left(\mathrm{mm}^{3}\right)$ or per unit lung surface area $\left(\mathrm{mm}^{2}\right)$ and related to age.

\section{Methods}

Twelve lifelong non-smokers (five men) with an age range of 21-78 years were studied. All had undergone surgical lobectomy $(n=10)$ or pneumonectomy $(n=2)$ for the investigation of peripheral lung opacities (six benign lesions, three primary lung carcinomas, three metastatic malignancies) between 1981 and 1990.

The specimens were transferred from

Department of Pathology, Edinburgh University Medical School, Teviot Place, Edinburgh EH8 9AG W A H Wallace M Gillooly

D Lamb

Reprint requests to: Dr W A H Wallace

Received 8 June 1992 Returned to authors 29 July 1992 stained with haematoxylin and eosin. Measurement of lung architecture was performed on the sections as previously described $^{2}$ with a fast interval processor (FIP). ${ }^{5}$ The results were expressed as airspace wall surface area per unit volume (AWUV; $\mathrm{mm}^{2} / \mathrm{mm}^{3}$ ). In this study AWUV was measured on all six stained sections from each case and the mean value obtained. Correction factors for shrinkage artifacts were not required due to the use of glycol methacrylate resin as the embedding medium. ${ }^{6}$

Alveolar macrophage numbers were counted at $250 \times$ magnification on 30 randomly selected $1 \mathrm{~mm}^{2}$ fields from the stained resin sections. We have previously observed that the distribution of alveolar macrophages in non-smokers tends to be more uniform than in smokers. Counting 30 fields per case was shown to give a running mean that did not vary by more than $5 \%$. For each case a mean macrophage score per $1 \mathrm{~mm}^{2}$ field was thus calculated. From this the alveolar macrophage number per $\mathrm{mm}^{3}$ lung volume and $\mathrm{mm}^{2}$ lung surface was calculated as described previously. ${ }^{2}$

Sections from each case had been stained previously with antisera to epithelial membrane antigen (EMA) to exclude significant pneumocyte desquamation into the alveolar space. In this study further sections were cut from tissue in the diagnostic archives for immunocytochemical staining with the antibody PC10 using a standard ABC method (Dako UK) to assess alveolar macrophage proliferation. ${ }^{7}$ Unfortunately PC10 was found not to be reactive on the older material which had been subjected to post-fixation processing with picric acid, so data were only available for four of the most recent cases.

\section{Results}

We found a significant positive correlation between increasing age and alveolar macrophage numbers whether this was expressed per $\mathrm{mm}^{3}$ lung volume (fig 1 : range

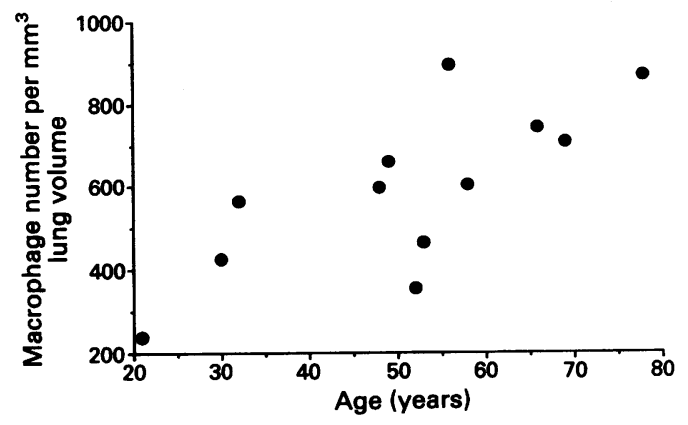

Figure 1 Alveolar macrophage number per $\mathrm{mm}^{3}$ lung volume with relation to age in 12 non-smoking patients (range 259.4-860.1: $r=0.735 ; p<0.01$ ). 
Figure 2 Alveolar macrophage number per $\mathrm{mm}^{2}$ lung surface area with relation to age in 12 non-smoking patients (range 11.9-43.6: $r=0.750 ; p<0.01$ ).

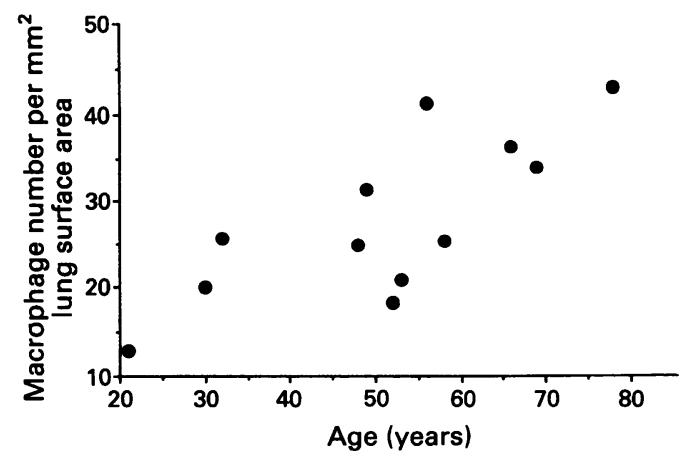

259.4-860.1; $r=0.735, p<0.01)$ or per $\mathrm{mm}^{2}$ lung surface area (fig 2: range 11.9-43.6; $r=0.750, \mathrm{p}<0.01)$.

Immunocytochemical staining with PC10 on the four available cases did not suggest any age related change in the proportion of alveolar macrophages proliferating (age(\%PC10 positive cells $\mathrm{n}>200)$ : $30(58)$; 32(41); 66(63); 78(43)).

\section{Discussion}

We have shown an age related increase in the alveolar macrophage population of nonsmokers with the use of a morphometric technique on tissue sections. We are unaware of any previous studies with bronchoalveolar lavage or other techniques which have shown this relationship. Using our approach we have shown that the increase in number can be expressed per unit lung volume or per unit lung surface area. The similarity of the two figures reflects the fact that non-smokers of any given age have similar AWUV values and similar numbers of alveolar macrophages. We believe that expressing the macrophage number in terms of alveolar wall surface area is more appropriate as the cells in vivo must relate to the surface of the lung. This also corrects for the known decrease in lung surface that occurs, even in non-smokers, with increasing age. ${ }^{34}$

The explanation for this observed relationship is not clear. No relationship between age and a malignant or benign diagnosis was apparent in the sample, benign lesions being present in some of the oldest and metastatic malignancy being present in the youngest.

It is known that there is no change in the human peripheral monocyte count ${ }^{8}$ or rat peritoneal macrophage number ${ }^{9}$ with increasing age. This suggests that the change in alveolar macrophage number may relate to unique conditions in the lung. It is possible that the increased numbers represent the cumulative effects of lifelong exposure to low level atmospheric irritants producing chronic low level injury. If this was the case one might expect to see differences between non-smokers from rural and urban environments as well as occupational differences. This would, however, require a larger study.

Another possible explanation is that the increase may be secondary to the ageing process in the lung which causes loss of lung surface area as measured by decreased AWUV. ${ }^{34}$ This change in lung architecture might interfere with the normal clearance pathways of alveolar macrophages and result in increased accumulation. The limited data gained from PC10 staining of the alveolar macrophages did not, however, suggest an accumulation of effete cells which might be expected if this was the case.

We conclude that the number of alveolar macrophages was increased with age in our study of 12 non-smokers. We have suggested two possible explanations for this observation. Unfortunately because of the difficulty in obtaining suitable surgical material from non-smokers the sample size in this study was small. Further study will be required to investigate the underlying mechanisms of this macrophage accumulation. We nevertheless report this as an interesting new observation.

1 Cherniak RM, and the BAL Cooperative Working Group. Bronchoalveolar lavage constituents in healthy individuals, idiopathic pulmonary fibrosis and selected comparison groups. Am Rev Respir Dis 1990;141(Suppl): parison grou

2 Wallace WAH, Gillooly $M$, Lamb $D$. Intra-alveolar macrophage numbers in current smokers and nonsmokers; a morphometric study of tissue sections. Thorax 1992;47:437-40.

3 Gillooly M, Lamb D. Airspace size in lungs of lifelong non-smokers: the effect of age and sex. Thorax 1993;48: 39-43

4 Gillooly M, Lamb D. Microscopic emphysema in relation to age and smoking habit. Am Rev Respir Dis 1992; 145:A762.

5 Gillooly M, Lamb D, Farrow ASJ. New automated technique for assessing emphysema on histological sections. nique for assessing emphysema

6 McLean A, Lamb D. Morphometry of small airways in man. F Pathol 1983;141:520.

7 Hall PA, Levison DA, Woods AL, Yu CC-W, Kellock $\mathrm{DB}$, Watkins JA, et al. Proliferating cell nuclear antigen (PCNA) immunolocalisation in paraffin sections: an index of cell proliferation with evidence of deregulated expression in some neoplasms. I Pathol 1990;162: 285-94.

8 Thomson JS, Wekstein DR, Rhoades JL. The immune status of healthy centenarians. $\mathcal{F}$ Am Geriatr Soc 1984; 32:274-81.

9 Makinodon T, Kay MMB. Age influence on the immune system. Adv Immunol 1980;29:287-98. 